\title{
Usefulness of bone turnover markers in the evaluation of fracture risk in type 2 diabetes
}

\author{
Pedro Rozas Moreno ${ }^{1,2}$, Rebeca Reyes García ${ }^{1,3}$, Antonia García Martín ${ }^{1,4}$, Manuel Muñoz Torres ${ }^{1}$. \\ ${ }^{1}$ Bone Metabolic Unit, Endocrinology Division, Hospital Universitario San Cecilio, Granada, Spain. \\ ${ }^{2}$ Endocrinology Division, Hospital General Universitario de Ciudad Real, Ciudad Real, Spain \\ ${ }^{3}$ Endocrinology Division, HGU Rafael Mendez, Lorca, Murcia. \\ ${ }^{4}$ Endocrinology Division, Hospital Comarcal del Noroeste, Caravaca, Murcia.
}

\section{INTRODUCTION:}

Although the contribution of high bone turnover to fracture risk is well-established, there is some controversy about the utility of the determination of bone turnover markers in the evaluation of fracture risk at the patient level. In type 2 diabetes (T2DM) there is an increased risk of fractures despite of higher bone mineral density. A recent study shows that a femoral neck T-score of -2.1 in males and -1.9 in females with T2DM present the same fracture risk than subjects without diabetes with a T-score -2.5 (Schwartz AV JAMA 2011).

\section{OBJECTIVES:}

To evaluate differences in bone turnover markers in patients with T2DM according to the presence of osteoporosis diagnosed by different classifications and FRAX index.

\section{METHODS:}

Cross-sectional study including 78 T2DM patients.

BMD was evaluated by DXA (Hologic QDR 4500).

Patients were classified as having or not osteoporosis according to WHO criteria, new criteria from Schwartz et al. and FRAX index.

We determined: bone-specific alkaline phosphatase (BSAP) (OCTEIATM IDS Ltd Boldon UK), osteocalcin (OC) (DiaSorin, Stillwater, Minnesota USA); TRA5b (Bone TRAP $®$ Assay. IDS Ltd); and CTX (Elecsys ß CrossLaps, Roche Diagnostics SL, Barcelona, Spain).

Results were analysed by SPSS 15.0 .

\section{RESULTS:}

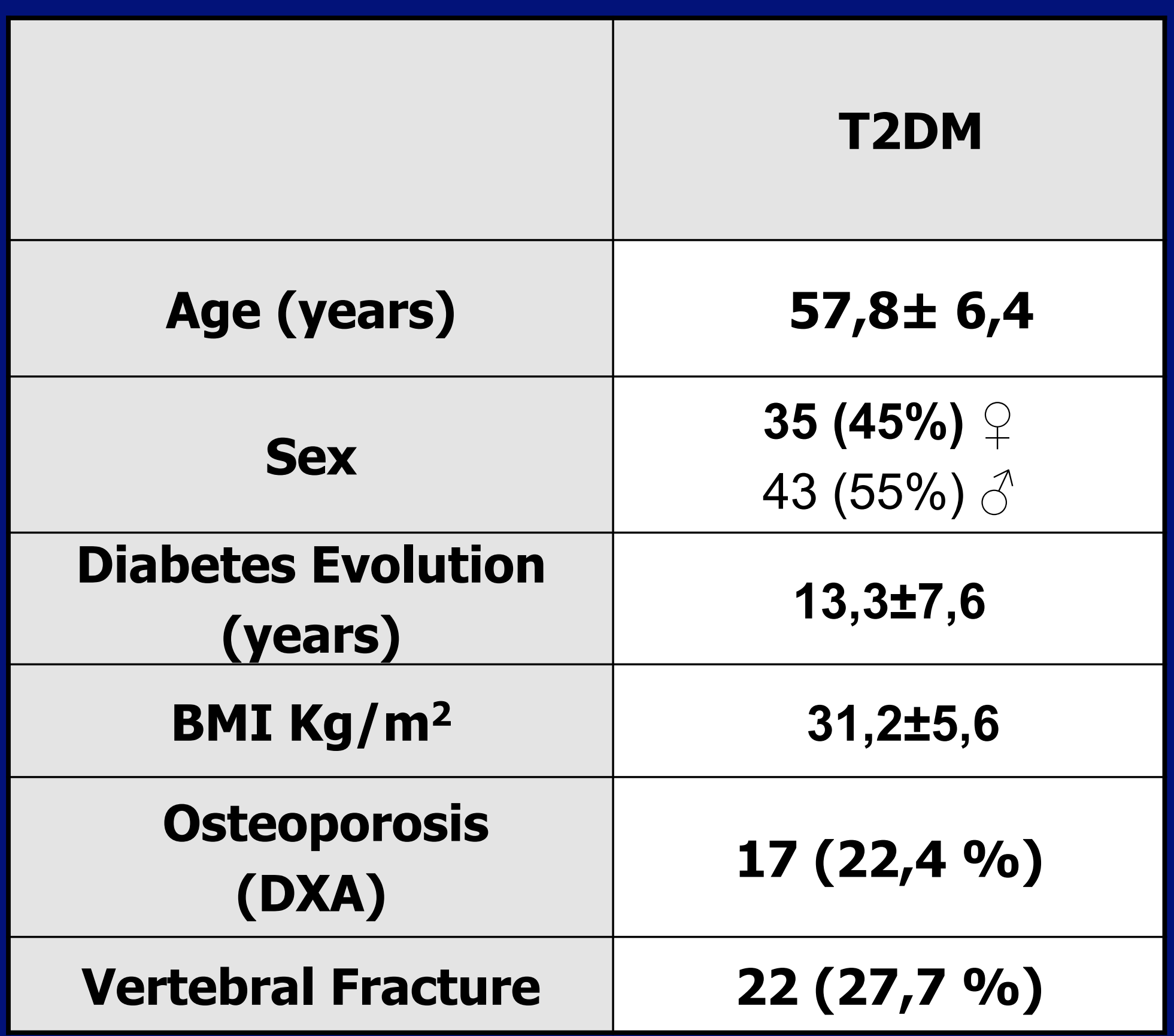

\section{BONE TURNOVER MARKERS ACCORDING TO THE THREE CLASSIFICATIONS}

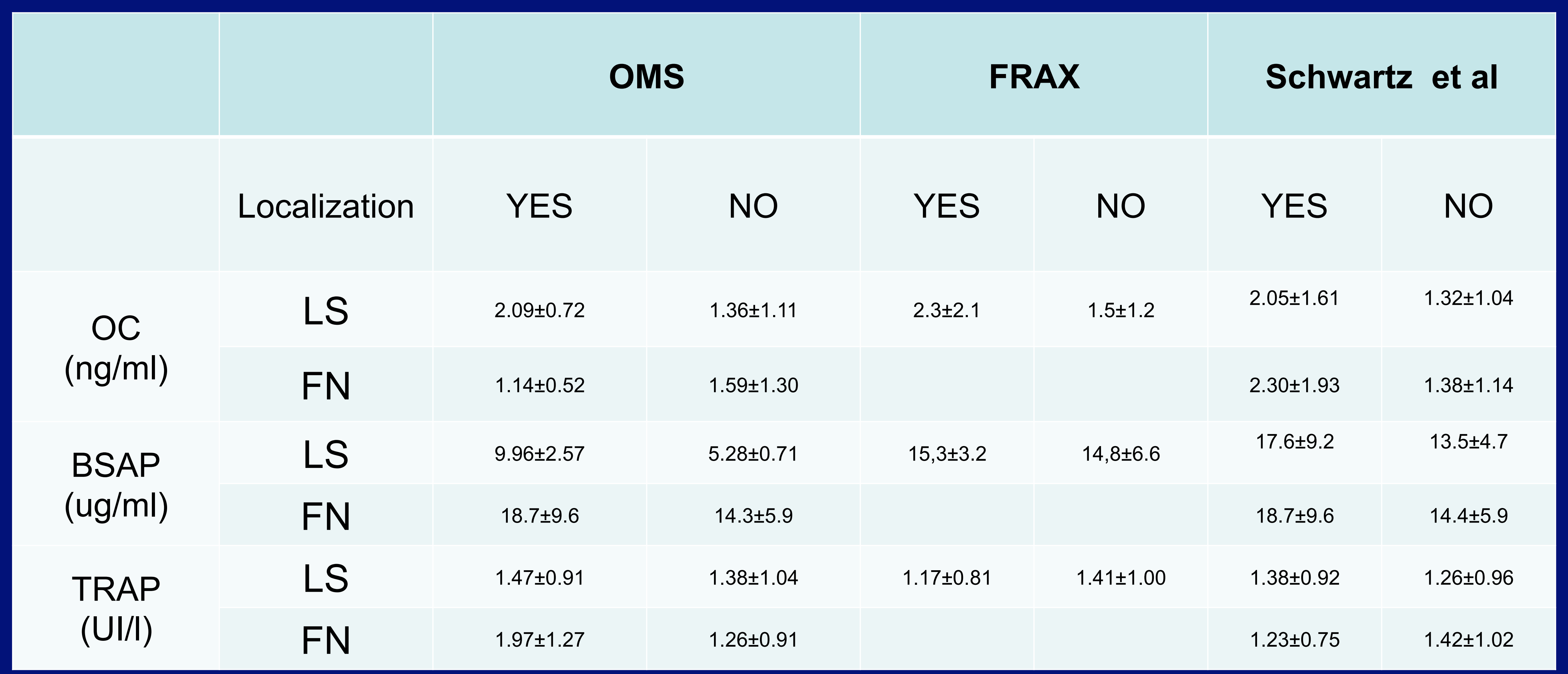

OC: osteocalcin; BSAP: bone-specific alkaline phosphatase; TRAP: tartate-specfic alkaline phosphatase LS: lumbar Spine; FE: femoral neck

CTX LEVELS (ng/ml) ACCORDING TO OSTEOPOROSIS CLASSIFICATION AND FRAX INDEX

SCHWARTZ ET AL

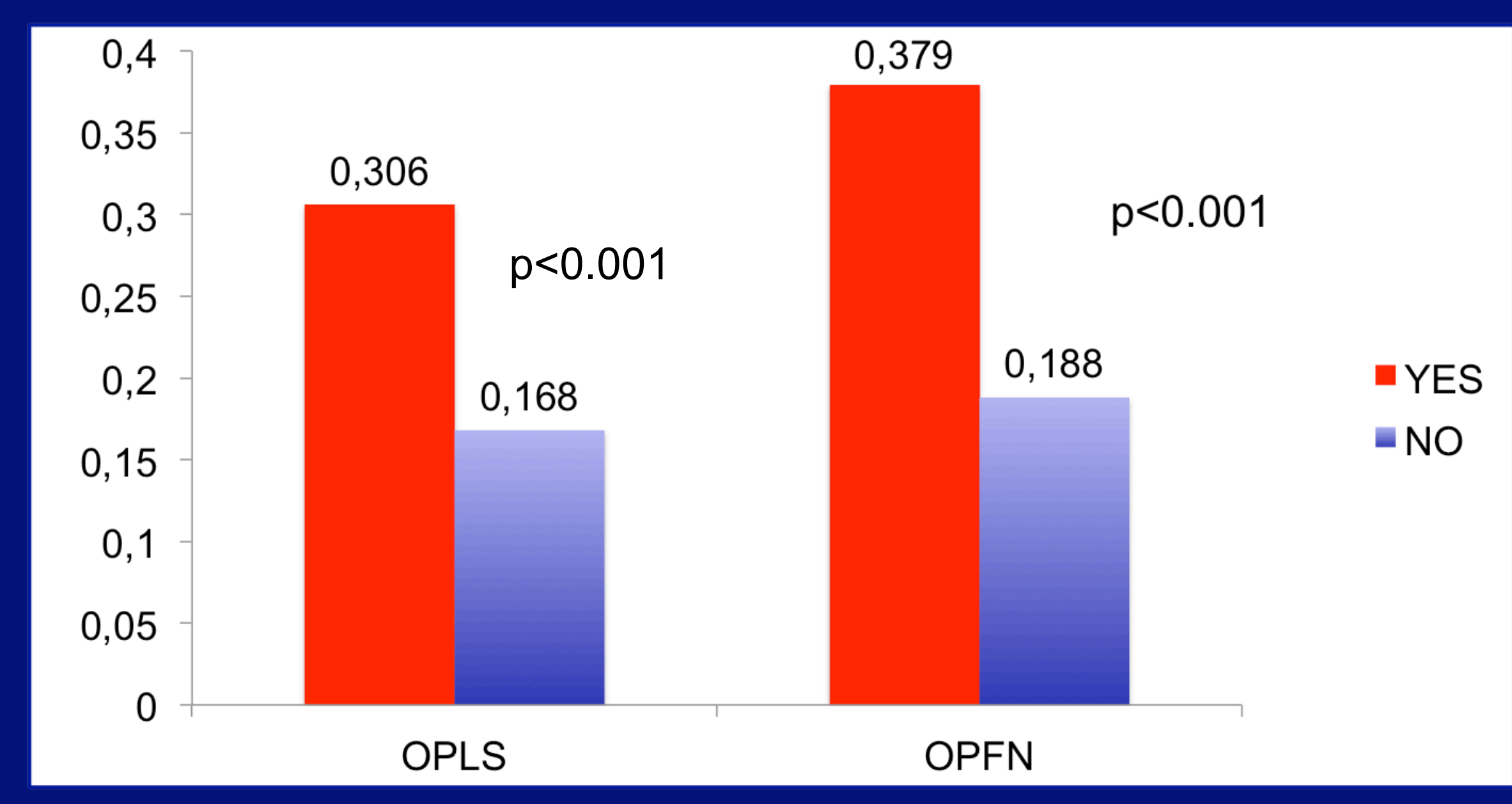

FRAX

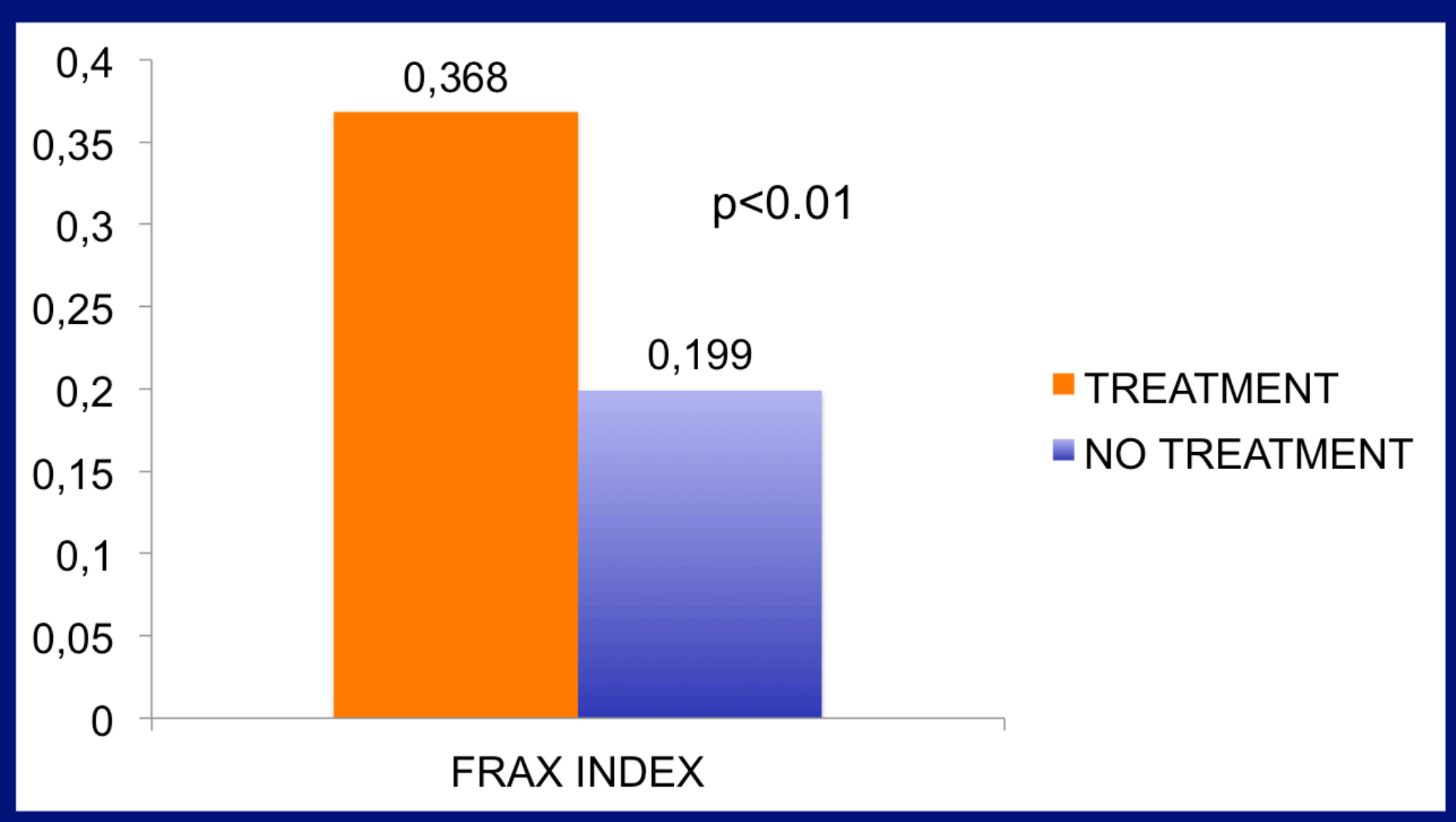

WHO

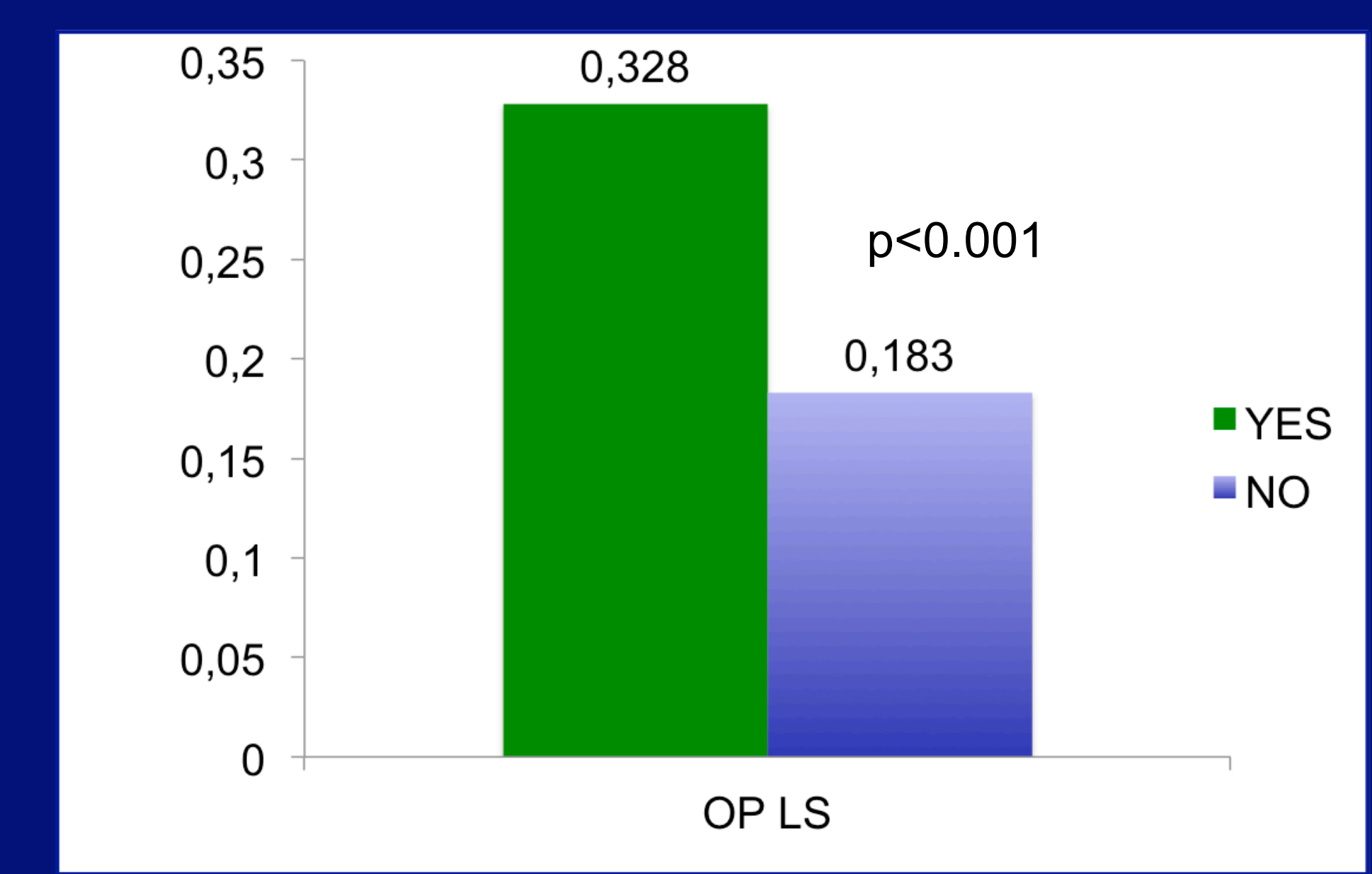

\section{CONCLUSIONS:}

Our results suggest that higher CTX concentrations may indicate which T2DM patients are suitable for osteoporosis treatment, although the measurement of CTX does not constitute a fracture risk factor independently of BMD. However, it may help in the identification of patients in whom DXA must be done. 